$04.2 ; 12.1$

\title{
Измерения спектров гамма- и нейтронного излучения в ядерных реакциях с ионами ${ }^{3} \mathrm{He}$ и ${ }^{9} \mathrm{Be}$
}

\author{
() М.В. Ильясова, А.Е. Шевелев, Е.М. Хилькевич, И.Н. Чугунов, Д.Н. Дойников, М.Ф. Кудояров, \\ B.О. Найденов, И.А. Полуновский
}

Физико-технический институт им. А.Ф. Иоффе РАН, Санкт-Петербург, Россия

E-mail: margaritavadimovna@gmail.com

Поступило в Редакцию 18 августа 2020 г.

В окончательной редакции 7 октября 2020 г.

Принято к публикации 15 октября 2020 г.

\begin{abstract}
Представлены результаты измерений спектров гамма- и нейтронного излучения, рожденного в ядерных реакциях ${ }^{9} \mathrm{Be}\left({ }^{3} \mathrm{He}, p \gamma\right){ }^{11} \mathrm{~B}$ и ${ }^{9} \mathrm{Be}\left({ }^{3} \mathrm{He}, n \gamma\right){ }^{11} \mathrm{C}$ при энергии ионов ${ }^{3} \mathrm{He} 2.57 \mathrm{MeV}$, с помощью органического детектора ВC-501А и полупроводникового спектрометра HPGe. Получены формы линий гамма-переходов $6.9 \mathrm{MeV}$ из реакции ${ }^{9} \mathrm{Be}\left({ }^{3} \mathrm{He}, n \gamma\right){ }^{11} \mathrm{C}$ и $8.92 \mathrm{MeV}$ из реакции ${ }^{9} \mathrm{Be}\left({ }^{3} \mathrm{He}, p \gamma\right){ }^{11} \mathrm{~B}$, измеренные под углами $0,30,60$, 90 и $120^{\circ}$. Восстановлено угловое распределение вылета протонов при заселении уровня $8.92 \mathrm{MeV}$ ядра ${ }^{11} \mathrm{~B}$ в реакции ${ }^{9} \mathrm{Be}\left({ }^{3} \mathrm{He}, p \gamma\right){ }^{11} \mathrm{~B}$, а также энергетическое распределение нейтронов в реакции ${ }^{9} \mathrm{Be}\left({ }^{3} \mathrm{He}, n \gamma\right){ }^{11} \mathrm{C}$.
\end{abstract}

Ключевые слова: нейтронная спектрометрия, гамма-спектрометрия, функции возбуждения, гамма-переход, диагностика высокотемпературной плазмы.

DOI: 10.21883/PJTF.2021.03.50565.18518

Одним из инструментов изучения энергетических распределений быстрых частиц в плазме токамака является гамма-спектрометрия. Гамма-диагностика основана на анализе спектров гамма-излучения, рождающегося во время разряда плазмы, с высоким энергетическим и временны́м разрешением в энергетическом диапазоне до $30 \mathrm{MeV}$ [1]. Источником гамма-излучения в разрядах плазмы являются ядерные реакции, протекающие между удерживаемыми быстрыми ионами и ионами примесей, например ионами $\mathrm{Be}$, а также ионами основной плазмы. Особую ценность диагностика быстрых частиц будет иметь при реализации будущих проектов, таких как ITER, так как процесс зажигания реакции термоядерного синтеза и поддержания горения плазмы невозможен без присутствия ускоренных частиц термоядерного топлива, а гамма-диагностика предоставляет инструменты для контроля и распределения быстрых частиц в плазме [2].

Для восстановления функций распределения быстрых частиц по энергии требуется информация о функциях возбуждения гамма-переходов ядер, рождающихся в реакциях с участием изучаемых ионов. Для получения энергетических распределений ускоренных изотопов ${ }^{3}$ Не методами гамма-спектрометрии требуется подробная информация о функциях гамма-переходов в ядерных реакциях ${ }^{9} \mathrm{Be}\left({ }^{3} \mathrm{He}, p \gamma\right){ }^{11} \mathrm{~B}$ и ${ }^{9} \mathrm{Be}\left({ }^{3} \mathrm{He}, n \gamma\right){ }^{11} \mathrm{C}$. В настоящее время наблюдается отсутствие полной информации о ядерных реакциях с участием ядер ${ }^{9} \mathrm{Be}$ и изотопа ${ }^{3} \mathrm{He}$, например отсутствуют детальные данные о функциях возбуждения гамма-переходов в указанных реакциях в широком диапазоне энергий налетающих частиц.

Кроме того, для анализа распределений быстрых ионов в плазме необходима информация об угловой зависимости функций возбуждения. В большинстве ис- следований угловая зависимость не изучалась, а измерения проводились при одном или двух направлениях вылета частиц-продуктов. Такие данные не позволяют установить интегральные сечения гамма-переходов, необходимые для получения функций энергетического распределения быстрых ионов в плазме токамака. Энергетические распределения быстрых ионов могут быть получены из анализа соотношения интенсивностей линий гамма-переходов, измеряемых спектрометрами в плазме [3], а также из анализа допплеровского уширения линий гамма-переходов $[4,5]$. В последнем случае в измерениях необходимо использовать полупроводниковый спектрометр HPGe с прецизионным энергетическим разрешением. Метод получения энергетического распределения быстрых ионов в плазме был разработан в ФТИ им. А.Ф. Иоффе $[4,5]$ и применен в экспериментах на токамаке JET [6,7].

Цель настоящей работы состоит в изучении ядерных реакций между ионами ${ }^{9} \mathrm{Be}$ и ${ }^{3} \mathrm{He}$ и дополнении уже имеющихся данных о функциях возбуждения гамма-переходов в данных реакциях и угловом распределении вылета частиц-продуктов в диапазоне $1-5 \mathrm{MeV}$. В рамках этого исследования на циклотроне ФТИ им. А.Ф. Иоффе были проведены измерения спектров гамма- и нейтронного излучения из бериллиевой мишени, облучаемой пучком ионов ${ }^{3} \mathrm{He}$ с энергией $2.57 \mathrm{MeV}$. В измерениях была использована бериллиевая мишень толщиной $111 \mu \mathrm{g} / \mathrm{cm}^{2}$. Бериллий был напылен на танталовую подложку толщиной $0.5 \mathrm{~mm}$.

Спектры гамма-излучения измерялись двумя спектрометрами HPGe: ORTEC GMX45P4-83-CW-PL с системой охлаждения X-Cooler II и CANBERRA GR5021 с электрическим кулером Cryo-Pulse 5 Plus. 
Относительная эффективность детектора ORTEC GMX45P4-83-CW-PL составляет 49\% (относительно эффективности $\mathrm{NaI}(\mathrm{Tl})$-детектора с $\varnothing 76.2 \times 76.2 \mathrm{~mm}$ при измерении гамма-квантов с энергией $1.3325 \mathrm{MeV}$ от источника на основе изотопа $\left.{ }^{60} \mathrm{Co}\right)$. Относительная эффективность CANBERRA GR5021 - 56.8\%. В ходе эксперимента положение детекторов менялось относительно направления пучка ионов, падающих на мишень. Это позволило измерить допплеровские формы линий в спектре гамма-квантов, соответствующих гамма-переходам с разной энергией, при углах обзора $0,30,60,90$ и $120^{\circ}$. Расстояние от мишени до детекторов составляло $40 \mathrm{~cm}$. На рис. 1 показаны формы линий в спектре гамма-квантов, соответствующих гамма-переходу $6.9 \mathrm{MeV}$ из реакции ${ }^{9} \mathrm{Be}\left({ }^{3} \mathrm{He}, n \gamma\right){ }^{11} \mathrm{C}$ и $8.92 \mathrm{MeV}$ из реакции ${ }^{9} \mathrm{Be}\left({ }^{3} \mathrm{He}, p \gamma\right){ }^{11} \mathrm{~B}$.

$\mathrm{B}$ измерениях спектров нейтронов из реакции $\left.{ }^{9} \mathrm{Be}\left({ }^{3} \mathrm{He}, n \gamma\right)\right)^{11} \mathrm{C}$ использовался спектрометр на основе жидкого органического сцинтиллятора ВС-501А производства компании Saint-Gobain с Ø50.8 $\times 50.8 \mathrm{~mm}$, соединенного с фотоэлектронным умножителем Hamamatsu R329-02. Измерение интенсивности дискретных линий в спектре нейтронного излучения позволяет получить парциальные сечения заселения уровней возбуждения ядра ${ }^{11} \mathrm{C}^{*}$, рожденного в реакции ${ }^{9} \mathrm{Be}\left({ }^{3} \mathrm{He}, n \gamma\right){ }^{11} \mathrm{C}$. Ней-

$a$

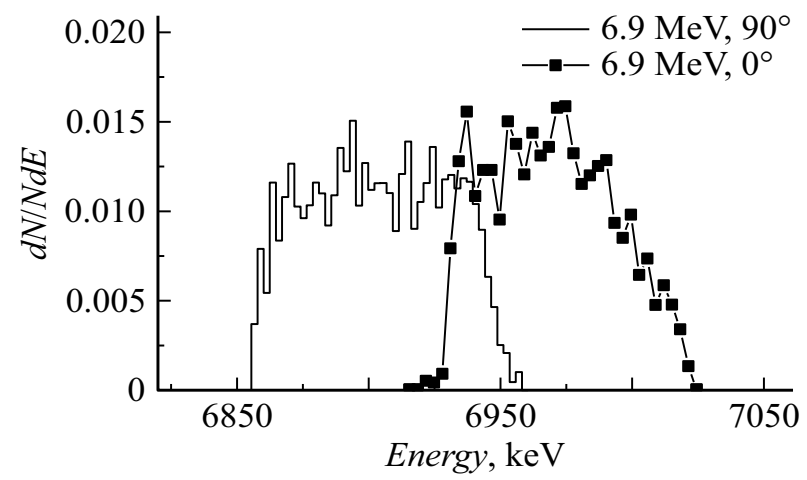

$b$

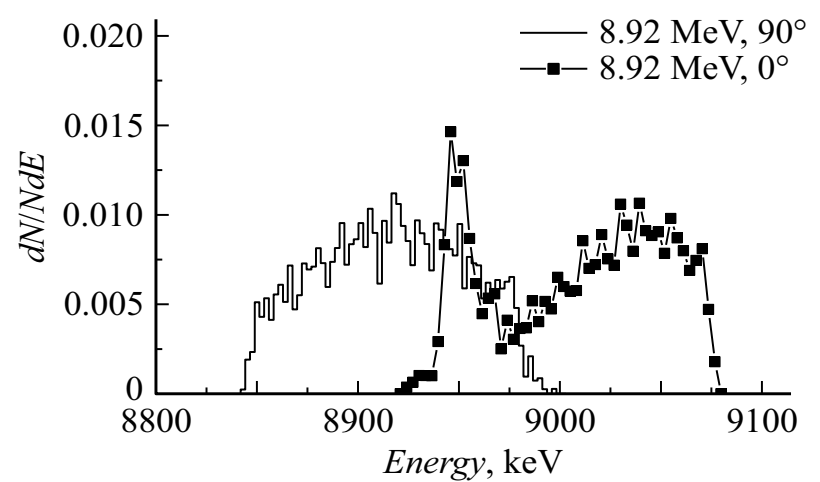

Рис. 1. Формы линий в спектре гамма-квантов, соответствующих переходу $6.9 \mathrm{MeV}$ из реакции ${ }^{9} \mathrm{Be}\left({ }^{3} \mathrm{He}, n \gamma\right){ }^{11} \mathrm{C}(a)$ и $8.92 \mathrm{MeV}$ из реакции ${ }^{9} \mathrm{Be}\left({ }^{3} \mathrm{He}, p \gamma\right)^{11} \mathrm{~B}(b)$, измеренные под углами 0 и $90^{\circ}$.

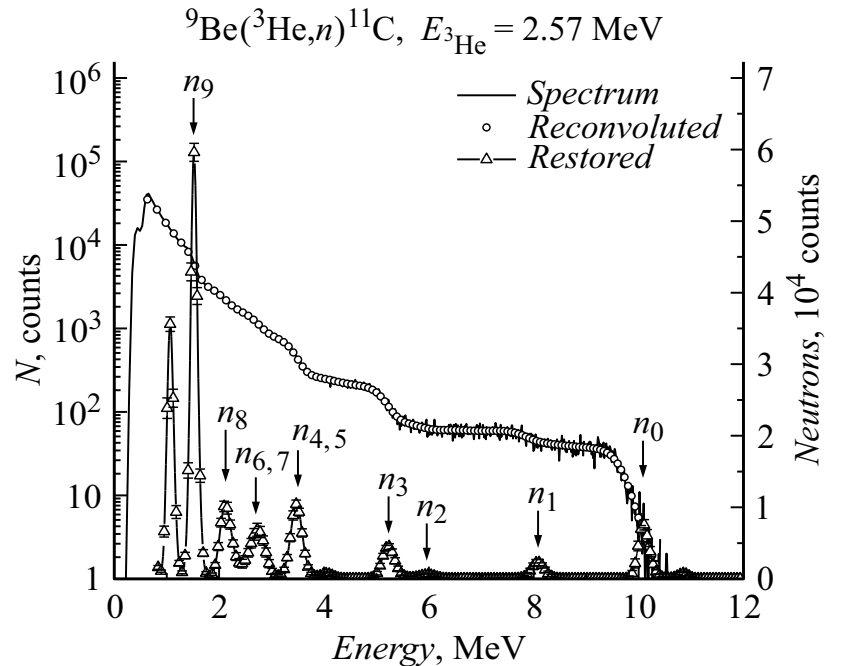

Рис. 2. Энергетический спектр нейтронов, рожденных в реакции ${ }^{9} \mathrm{Be}\left({ }^{3} \mathrm{He}, n \gamma\right){ }^{11} \mathrm{C}$, измеренный нейтронным спектрометром BC-501А под углом $15^{\circ}$ относительно направления пучка ${ }^{3} \mathrm{He}$ с энергией $2.57 \mathrm{MeV}$ (сплошная линия). Треугольники восстановленное энергетическое распределение нейтронов, кружки - обратная свертка восстановленного распределения нейтронов с функциями отклика спектрометра; $n_{0}, n_{1}, n_{2}, n_{3}, n_{4}$, $n_{5}, n_{6}, n_{7}, n_{8}, n_{9}$ - энергетические группы нейтронов с энергией $10.06,8.08,5.75,5.27,3.69,3.54,3.16,2.56,1.96,1.64 \mathrm{MeV}$ соответственно, испускаемые ядром ${ }^{12} \mathrm{C}^{*}$ и заселяющие уровни возбуждения ядра ${ }^{11} \mathrm{C}$.

тронный спектрометр прошел калибровку на пучке циклотрона ФТИ им. А.Ф. Иоффе, в результате чего был получен набор функций отклика детектора на моноэнергетическое нейтронное излучение в диапазоне энергий $1.9-10.4 \mathrm{MeV}$, а также энергетическая зависимость эффективности регистрации нейтронов [8]. На рис. 2 показано энергетическое распределение нейтронов, вылетающих из мишени под углом $15^{\circ}$ относительно направления пучка ${ }^{3} \mathrm{He}$, полученное после обработки измеренного спектра кодом DeGaSum [8] с использованием функций отклика спектрометра.

Анализ допплеровской формы линий в спектре гамма-квантов позволил восстановить угловое распределение продуктов реакций (нейтронов и протонов), проходящих в бериллиевой мишени при облучении пучком ${ }^{3} \mathrm{He}$. Поиск углового распределения продуктов находился методом, описанным в работе [5].

Исследуемые реакции имеют двухступенчатый характер типа $A(a, b) B(\gamma) C$, и амплитуда этих реакций факторизуется на амплитуду реакции $A(a, b) B$ и амплитуду гамма-распада. Угловую зависимость вероятности испускания частицы-продукта реакции $b$ в системе центра масс относительно направления налетающего ядра ${ }^{3} \mathrm{He}$ можно выразить через полиномы Лежандра $P_{k}\left(\cos \theta_{b}\right)$

$$
w\left(\theta_{b}\right)=\alpha_{0} \sum_{k=1}\left[1+\frac{\alpha_{k}}{\alpha_{0}} P_{k}\left(\cos \theta_{b}\right)\right]
$$


и использовать для описания формы линии. В формуле (1) $\alpha_{k}$ - численные коэффициенты, $\theta_{b}$ - угол испускания частицы $b$ в системе центра масс. Энергетическое распределение гамма-квантов в спектре, наблюдаемом под углом $0^{\circ}$ относительно направления пучка ${ }^{3} \mathrm{He}$, будет иметь вид (с учетом искажений, вносимых конечным разрешением детектора)

$$
\begin{aligned}
S\left(E_{\gamma}\right)= & \alpha_{0} \int_{E_{\gamma}^{\min }}^{E_{\gamma}^{\max }} d \varepsilon_{\gamma} \sum_{k=1}\left[1+\frac{\alpha_{k}}{\alpha_{0}} P_{k}\left(\cos \theta_{b}\right)\right] \\
& \times \frac{1}{\sigma \sqrt{2 \pi}} \exp \left[-\frac{\left(E_{\gamma}-\varepsilon_{\gamma}\right)^{2}}{2 \sigma^{2}}\right] .
\end{aligned}
$$

Здесь энергия $E_{\gamma}$ гамма-излучения, испускаемого ядрами отдачи, равна

$$
E_{\gamma}=E_{\gamma 0}\left(1+\frac{V_{c m}}{c}-\frac{V_{B}}{c} \cos \theta_{b}\right)
$$

и, таким образом, связана с углом $\theta_{b}$ испускания частицы $b$ в системе центра масс. $V_{c m}-$ скорость движения центра масс (компаунд-ядра), $V_{B}$ - скорость ядра отдачи (после вылета легкой частицы) в системе центра масс, $c$ - скорость света.

Энергетическое разрешение спектрометра учитывается в форме распределения Гаусса со стандартным отклонением, равным $\sigma$. Пределы интегрирования $\left(E_{\gamma}^{\min }, E_{\gamma}^{\max }\right)$ соответствуют предельным значениям $\cos \theta_{n}=(1,-1)$. Коэффициенты разложения $\alpha_{k}$ функции углового распределения частицы $b$ можно найти путем подгонки расчетной функции $S\left(E_{\gamma}\right)$ к экспериментально измеренной форме линии $S^{\exp }\left(E_{\gamma}\right)$, минимизируя функционал

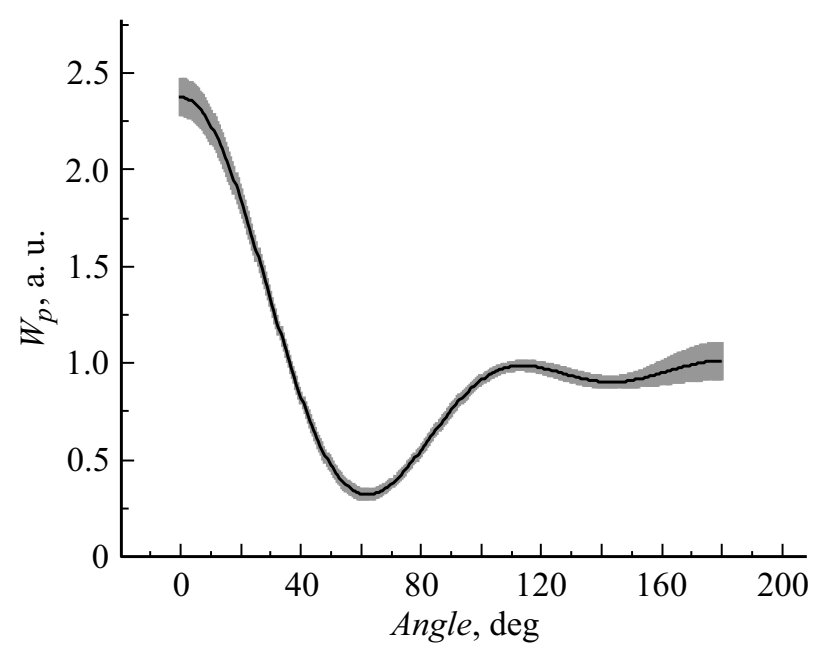

Рис. 3. Угловое распределение вылета протонов при заселении уровня $8.92 \mathrm{MeV}$ ядра ${ }^{11} \mathrm{~B}$ в реакции ${ }^{9} \mathrm{Be}\left({ }^{3} \mathrm{He}, p \gamma\right){ }^{11} \mathrm{~B}$ относительно направления пучка ${ }^{3} \mathrm{He}$ при энергии ${ }^{3} \mathrm{He} 2.57 \mathrm{MeV}$. Неопределенности нахождения углового распределения отмечены серым.
$\left\|S^{\exp }\left(E_{\gamma}\right)-S\left(E_{\gamma}\right)\right\|$ относительно значений $\alpha_{k}$. На рис. 3 показано угловое распределение вылета протонов при заселении уровня $8.92 \mathrm{MeV}$ ядра ${ }^{11} \mathrm{~B}$ в ходе реакции ${ }^{9} \mathrm{Be}\left({ }^{3} \mathrm{He}, p \gamma\right){ }^{11} \mathrm{~B}$ относительно направления пучка ${ }^{3} \mathrm{He}$.

В ходе эксперимента по облучению бериллиевой мишени пучком ${ }^{3} \mathrm{He} \mathrm{c} \mathrm{энергией} 2.57 \mathrm{MeV}$ были измерены спектры нейтронного и гамма-излучения. Анализ допплеровски уширенной линии в спектре гамма-квантов, соответствующих переходу $8.92 \mathrm{MeV}$ ядра ${ }^{11} \mathrm{~B}$ в реакции ${ }^{9} \mathrm{Be}\left({ }^{3} \mathrm{He}, p \gamma\right){ }^{11} \mathrm{~B}$, позволил восстановить угловое распределение вылета протонов. Форма углового распределения отражает суперпозицию механизмов прямой ядерной реакции и реакции, протекающей через образование составного ядра. Вклад этих механизмов требует дальнейшего детального изучения.

Использованная аппаратура и методики будут применяться при изучении функций возбуждения и допплеровских форм линий в гамма-спектрах в ядерных реакциях ${ }^{9} \mathrm{Be}\left({ }^{3} \mathrm{He}, p \gamma\right){ }^{11} \mathrm{~B}$ и ${ }^{9} \mathrm{Be}\left({ }^{3} \mathrm{He}, n \gamma\right){ }^{11} \mathrm{C}$ в энергетическом диапазоне $1-5 \mathrm{MeV}$.

\section{Благодарности}

Авторы выражают благодарность В.Г. Киптилому (CCFE, Абингдон, Великобритания) за консультирование в ходе анализа экспериментальных данных.

\section{Финансирование работы}

Исследование выполнено при финансовой поддержке Российского фонда фундаментальных исследований в рамках научного проекта № 19-32-90081.

\section{Конфликт интересов}

Авторы заявляют, что у них нет конфликта интересов.

\section{Список литературы}

[1] V.G. Kiptily, F.E. Cecil, S.S. Medley, Plasma Phys. Control. Fusion, 48, R59 (2006).

[2] V. Kiptily, Ye. Kazakov, M. Fitzgerald, M. Nocente, M. Iliasova, E. Khilkevich, M.J. Mantsinen, M.F.F. Nave, J. Ongena, S.E. Sharapov, A.E. Shevelev, Ž. Štancar, G. Szepesi, D.M.A. Taylor, Yu.V. Yakovenko and JET Contributors, Nucl. Fusion, 60, 112003 (2020).

[3] A.E. Shevelev, E.M. Khilkevitch, V.G. Kiptily, I.N. Chugunov, D.B. Gin, D.N. Doinikov, V.O. Naidenov, A.E. Litvinov, I.A. Polunovskii and JET-EFDA Contributors, Nucl. Fusion, 53, 123004 (2013).

[4] V.G. Kiptilyj, A.V. Matjukov, A.S. Mishin, V.O. Najdenov, I.A. Polunovskij, L.A. Rassadin, I.N. Chugumov, Fusion Technol., 22, 454 (1992).

[5] В.Г. Киптилый, Д.Н. Дойников, В.О. Найденов, И.А. Полуновский, И.Н. Чугунов, Вопр. атомной науки и техники. Сер. Физика ядерных реакторов. Специальный выпуск, ТИЯС-XI, 223 (1997). 
[6] V.G. Kiptily, G. Gorini, M. Tardocchi, P.C. de Vries, F.E. Cecil, I.N. Chugunov, T. Craciunescu, M. Gatu Johnson, D. Gin, V. Goloborod'ko, C. Hellesen, T. Johnson, K. Kneupner, A. Murari, M. Nocente, E. Perelli, A. Pietropaolo, S.D. Pinches, I. Proverbio, P.G. Sanchez, S.E. Sharapov, A.E. Shevelev, D.B. Syme, V. Yavorskij, V.L. Zoita and JET-EFDA Contributors, Nucl. Fusion, 50, 084001 (2010).

[7] M. Nocente, M. Tardocchi, V.G. Kiptily, P. Blanchard, I. Chugunov, S. Conroy, T. Edlington, A.M. Fernandes, G. Ericsson, M. Gatu Johnson, D. Gin, G. Grosso, C. Hellesen, K. Kneupner, E. Lerche, A. Murari, A. Neto, R.C. Pereira, E. Perelli Cippo, S. Sharapov, A. Shevelev, J. Sousa, D.B. Syme, D. Van Eester, G. Gorini and JET-EFDA Contributors, Nucl. Fusion, 52, 063009 (2012).

[8] M.V. Iliasova, A.E. Shevelev, E.M. Khilkevitch, I.N. Chugunov, V.B. Minaev, D.B. Gin, D.N. Doinikov, I.A. Polunovsky, V.O. Naidenov, M.A. Kozlovskiy, M.F. Kudoyarov, Nucl. Instrum. Meth. Phys. Res. A, 983, 164590 (2020). 